\title{
Upregulation of RASSF1A in Colon Cancer by Suppression of Angiogenesis Signaling and Akt Activation
}

\author{
Thomas G. Blanchard ${ }^{\mathrm{a}} \quad$ Rena Lapidus ${ }^{\mathrm{b}} \quad$ Vivekjyoti Banerjee $^{\mathrm{a}} \quad$ Andrea C. Bafford $^{\mathrm{c}}$ \\ Steven J. Czinn ${ }^{\mathrm{a}}$ Hafiz Ahmed $^{\mathrm{d}}$ Aditi Banerjee $^{\mathrm{a}}$ \\ aDepartment of Pediatrics, University of Maryland School of Medicine, Baltimore, Maryland, \\ bDepartment of Medicine, University of Maryland School of Medicine, Baltimore, Maryland, \\ 'Department of Surgery, University of Maryland School of Medicine, Baltimore, MD, dGlycoMantra Inc, \\ Baltimore, Maryland, U.S.A
}

\section{Key Words \\ Andrographolide (AGP) • RASSF1A • PTEN • ER stress • Angiogenesis • Colon Cancer}

\begin{abstract}
Background/Aims: Silencing of tumor suppressor genes (TSGs) and promotion of angiogenesis are associated with tumor development and metastasis. However, little is known if angiogenic molecules directly control TSGs and vice versa. Methods: A regulatory link between angiogenesis and down regulation of TSGs was evaluated using an anti-cancer agent, andrographolide (AGP) in cancer cells, mouse xenograft tissues and patient derived organoids through gene/protein expression, gene silencing, and immunohistochemical analyses. Results: AGP treatment demonstrated significant expression of RASSF1A and PTEN TSGs in colon cancer and other cancer cells, mouse tissues and organoids. Depletion of RASSF1A with siRNA limited cyclin D1 and BAX expression. SiRNA depletion of PTEN, upstream regulator of RASSF1A resulted in a 50\% reduction in RASSF1A expression. Histopathological analysis of the AGP treated tumor sections showed significant reduction in vessel size, microvascular density and tumor mitotic index suggesting suppression of angiogenesis. This was corroborated by protein analysis demonstrating significant reductions in angiogenesis signaling pathway molecules $\mathrm{VEGF}_{165}$ FOXM1, and pAkt, but significant elevation of the endogenous angiogenesis inhibitor Tsp-2. Treatment of cells with exogenous VEGF prevented the suppression of angiogenesis signaling by AGP, resulting in sustained expression of pAkt, an upstream down-regulator of RASSF1A. RASSF1A expression remained low in VEGF treated cells despite the addition of AGP. Conclusion: Our results demonstrate for the first time that AGP induces RASSF1A expression in colon cancer cells and is dependent on angiogenesis signaling events. Therefore, our research may facilitate novel therapeutic options for advanced colon cancer therapy.




\section{Introduction}

Initiation of cancer progression is due to an imbalance of activation of cellular oncogenes and the loss of function of tumor suppressor genes (TSG) [1]. One such TSG is the Rasassociation domain family A (RASSFA) [2,3]. RASSF1A is subject to epigenetic regulation and suppression of RASSF1A is largely mediated through methylation. A previous study reported RASSF1A methylation ranging from $12 \%$ to $81 \%$ in colorectal cancer (CRC) cells [4, 5]. The biological functions of RASSF1A are still being elucidated. Several studies have demonstrated that the RASSF1A isoform is mainly involved in cell cycle control and DNA repair [6, 7]. Other studies have demonstrated its role on growth regulation and pro-apoptotic pathways [8-13].

In addition to the genetically controlled development of tumor cells, tumor angiogenesis also plays a critical role in tumor development. The potent and prevalent proangiogenic factor VEGF-A is a primary driver of angiogenic signaling. It signals through its receptors, primarily VEGFR2.The upregulation of this receptor in tumor cells is due to the activation of oncogenes and inactivation of tumor suppressor genes during neoplastic transformation. After binding with its ligand, this receptor responds by signaling through the Raf-MEK-ERK and PI3K-Akt kinase cascades [14, 15]. Novel therapeutic agents that target angiogenic mechanisms would serve as a valuable adjunct to treat cancer including colon cancer.

We have previously demonstrated that the plant metabolite andrographolide (AGP) induces cell death in CRC cells and that this activity is dependent on the activation of IRE-1, an ER stress marker [16]. We now report that AGP enhances RASSF1A expression in CRC cells and colon cancer tumor tissue and that this activity is also dependent on ER stress. In our model, depletion of RASSF1A during AGP treatment resulted in reduced apoptosis signaling and arrest of the cell cycle. We also demonstrate that AGP inhibits angiogenesis through the downregulation of pro-angiogenic factors including VEGF ${ }_{165}$; VEGFR2, FOXM1, and PTTG1. Notably, suppression of angiogenesis signaling suppresses Akt activation, a critical event in the upregulation of RASSF1A expression. This is the first report that cancer treatment suppressing angiogenesis promotes expression of RASSF1A and other tumor suppressor genes.

\section{Materials and Methods}

\section{Ethics Statement}

De-identified colon cancer tissues were collected from patients undergoing colorectal surgery with the approval of the University of Maryland Institutional Review Board (HP-00066889). Written consent was obtained from all patients from whom discarded tissue was collected which included permission to publish results. Animal experiments were performed in accordance with the N.I.H. Guide for the Care and Use of Laboratory Animals. The protocol was approved by the Institutional Animal Care and Use Committee of the University of Maryland in Baltimore (1113007).

\section{Cell culture and AGP treatment}

T84, Colo 205 and HCT 116 colon cancer cell lines were cultured and treated with AGP, 4PBA or TM at the indicated concentrations as previously described $[16,17]$. To evaluate the relationship between AGP induced suppression of angiogenesis signaling and RASSF1A expression, the colon cancer cells were stimulated with VEGF ${ }_{165}(10 \mathrm{ng} /$ well $)$ for 10 mins and then treated with AGP for $48 \mathrm{~h}$. RASSF1A expression and VEGF associated signaling events were monitored by western blot.

\section{Generation and propagation of patient-derived organoid cell cultures}

Organoid cell cultures were established based on the method of Sato et al. with slight modifications [18]. Briefly, resected colon carcinoma tissue was rinsed with cold PBS and tissue fragments $\left(\sim 2 \mathrm{~mm}^{3}\right)$ were digested with collagenase IV (Sigma-Aldrich), DNaseI (Applichem), and dispase (Stem Cell Technologies) in advanced DMEM/F12 (Life Technologies) supplemented with $1 \mathrm{X}$ penicillin/streptomycin at $37^{\circ} \mathrm{C}$ for 1 h. Fragments were recovered by centrifugation at $300 \mathrm{~g}$ for $3 \mathrm{~min}$ and resuspended in Advanced DMEM/ F12 with antibiotics. The suspension was filtered through $100 \mu \mathrm{m}$ and then $40 \mu \mathrm{m}$ cell strainers and 


\section{Cellular Physiology Cell Physiol Biochem 2018;48:1259-1273 and Biochemistry Published online: July 25, $2018 \quad \begin{aligned} & \text { DOI: 10.1159/000492012 } 2018 \text { The Author(s). Published by S. Karger AG, Basel } \\ & \text { www.karger.com/cpb }\end{aligned}$ \\ Blanchard et al.: RASSF1A Regulation by Angiogenic Signal}

recovered by centrifugation. Cell fractions were mixed with phenol-free growth factor-reduced Matrigel (Corning) and seeded in 24- well plates as $20 \mu \mathrm{l}$ domes. Matrigel domes were then overlaid with $500 \mu \mathrm{l}$ of Advanced DMEM supplemented with glutamax, penicillin/streptomycin, 1X N2 (Gemini BioProducts), 1X B27 (Gemini BioProducts), 1mM N-acetyl-L-cysteine (sigma Aldrich), $20 \mathrm{ng} / \mathrm{ml}$ fibroblast growth factor (Gemini BioProducts), and $50 \mathrm{ng} / \mathrm{ml}$ epidermal growth factor (Gemini BioProducts). The cultures were passaged when the aggregates reached a diameter of approximately $800 \mu \mathrm{m}$. Organoids were passaged by trypsinization and replated in a 24 well plates at 1:3 dilution.

\section{Endothelial cell culture and viability assay}

Endothelial cells (HUVEC) were a gift from Joshua Lewis, Ph.D. (University of Maryland School of Medicine). Cells were cultured as previously published methods [19]. Cell viability in the presence or absence of AGP was assessed using the MTT assay as previously described [20].

\section{Immunoblotting}

Immunoblotting were performed as previously described [21]. The primary antibodies used were against RASSF1A (eBioscience, San Diego, CA, 14-6888-80), p-YAP (Cell signaling, Ser127, D9W21), p-Akt (thr308) (Cell signaling, 9275), IRE-1 (Cell signaling, 3294), YAP (G-6) (sc-376830, Santa Cruz Biotechnology, Dallas, TX), phospho-VEGFR1/Flt-1 (Y1213) (AF4170), phospho-VEGFR2 /KDR/Flk-1 (Y1214) (AF1766, R\&D systems), FOXM1 (R\&D systems, Minneapolis, MN). Images were captured using a Syngene G Box digital imager (Frederick, MD) and results were quantified by densitometry as previously described [21].

\section{Quantitative real-time polymerase chain reaction ( $q R T-P C R$ )}

Gene expression was evaluated as previously described [16]. Primer sequences are listed (for all online suppl. material, see www.karger.com/doi/10.1159/000492012) in Suppl. Table 1. Relative gene expression changes were calculated using the 2- $\Delta \Delta \mathrm{CT}$ method, and expression normalization was accomplished using housekeeping gene GAPDH.

\section{Manipulation of RASSF1A, PTEN and IRE-1 expression}

Depletion studies were performed using siRNA specific for RASSF1A, PTEN and IRE-1 and a proprietary universal negative control siRNA (Sigma Aldrich, \#EHU088291, \#EHU106441, \#EHU002721, and \#SIC001 respectively) [16]. The siRNA reagents are a mixture of siRNA sequences prepared from an enzymatic digestion cDNA. T84 and Colo 205 cells were transfected with siRNA using TurboFect (ThermoFisher Scientific, \#R0533). Briefly, T84 cells ( $5 \times 10^{4}$ cells/well) and for Colo 205 cells ( $5 \times 10^{5}$ cells /well) in 24 well plates were transfected with $1 \mu \mathrm{g}$ siRNA in $100 \mu \mathrm{l}$ serum free DMEM and transfection reagent and cells were analyzed for gene expression at 24 and $48 \mathrm{~h}$. For activation experiments, transfected cells were treated with AGP for $48 \mathrm{~h}$. Gene depletions were validated by qRT-PCR. IRE-1 overexpression was performed as previously described using a plasmid for IRE-1 overexpression (IRE-1 $\alpha$-pcDNA3.EGFP, \#13009) purchased from Addgene (Cambridge, MA) [16].

\section{Mouse xenograft model}

Athymic Balb/c (nu/nu) mice aged 6 weeks were housed under pathogen-free conditions in micro isolator cages at the University of Maryland Baltimore animal center. Groups of five to seven mice were injected in the flank with 5X10 $0^{6}$ HCT 116 cells to induce tumor development. Mice were treated with AGP either by injection (IP) or orally (PO) at a dose of $50 \mathrm{mg} / \mathrm{kg}$ body weight when tumors reached 400-500 $\mathrm{mm}^{3}$. Mice were dosed for five consecutive days. Mice were euthanized either 1 hour or 4 hours post-dosing following the final AGP treatment. Tumors were excised and weighed and then half of each tumor was fixed in $10 \%$ buffered formalin and the other half was frozen for subsequent analysis.

\section{Histological staining and Microvessel Counts (MVC)}

Formalin fixed HCT 116 tumor tissue was embedded in paraffin and $4 \mu \mathrm{M}$ sections were stained with $\mathrm{H} \& \mathrm{E}$ and analyzed microscopically to quantify the mitotic index using the ratio of cell numbers undergoing mitosis (any stage of cell division) to the number of cells not undergoing cell division. The MVC were analyzed as previously described [22]. Five hot spots from each group were evaluated under 200X magnification and microscopic images from each area were collected (ASI C-203) using an Olympus BX53 with the use of bioquant lifescience 2017 program and recorded as MVC.

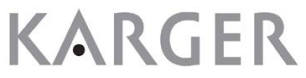




\section{Cellular Physiology Cell Physiol Biochem 2018;48:1259-1273 \begin{tabular}{l|l} 
and Biochemistry Published online: July 25, 2018 & $\begin{array}{l}\text { (c) } 2018 \text { The Author(s). Published by S. Karger AG, Basel } \\
\text { www.karger.com/cpb }\end{array}$
\end{tabular} \\ Blanchard et al.: RASSF1A Regulation by Angiogenic Signal}

\section{Immunohistochemistry from tumor tissue}

Proliferative differences between control and AGP-treated HCT 116 tumor tissues were determined by immunohistochemical staining for Ki67 as previously described [23]. Tissue sections were incubated with anti-Ki67 (M-19) antibody (Santa Cruz \# sc-7846) followed by ImmPRESS reagents for goat antibody (MP7405, Vector Laboratories, Burlingame, CA) and development with brown ImmPACT DAB HRP substrate (Vector). Images were captured as described earlier (23).

\section{Statistical analyses}

Statistical analysis was performed with Graph Pad Prism for Macintosh 5.0c (Graph Pad Software Inc., San Diego, CA). The mean S.E. was calculated by one way ANOVA. Significance between groups was analyzed using the post hoc Tukey's test and Bonferroni test. P values were considered significant if less than 0.05 and are indicated throughout using asterisks ${ }^{*}=\mathrm{P}<0.05,{ }^{* *}=\mathrm{P}<0.01,{ }^{* * *}=\mathrm{P}<0.001$.

\section{Results}

AGP alters expression of tumor suppressor genes in CRC cells

Many TSGs are associated with progression of the malignant state in CRC [1]. We evaluated TSG expression in CRC cell lines (T84, Colo 205 and HCT 116 cells) to determine if AGP treatment influences their expression. Cells were treated with AGP $\left(\mathrm{IC}_{50}=45 \mu \mathrm{M}\right)$ for 48 $\mathrm{h}$ and gene expression was evaluated by qRT-PCR (Fig. 1). Expression of RUNx, MLH, and HPP1 were uniformly suppressed and APC was unaffected (see online suppl. material, Suppl. Fig.1 A-E). Conversely, CDKN2A, PTEN and RASSF1A expression was significantly upregulated in T84 and Colo 205 cells (Fig. $1 \mathrm{~A}, \mathrm{~B}$, and $\mathrm{C}, P<0.05$ ). RASSF1A expression was also upregulated in HCT 116 cells $(P<0.01)$ and overall, RASSF1A was observed to exhibit the most pronounced increased in expression.

AGP induces RASSF1A expression in cells from multiple types of cancer

RASSF1A expression is suppressed in many types of cancer including colon

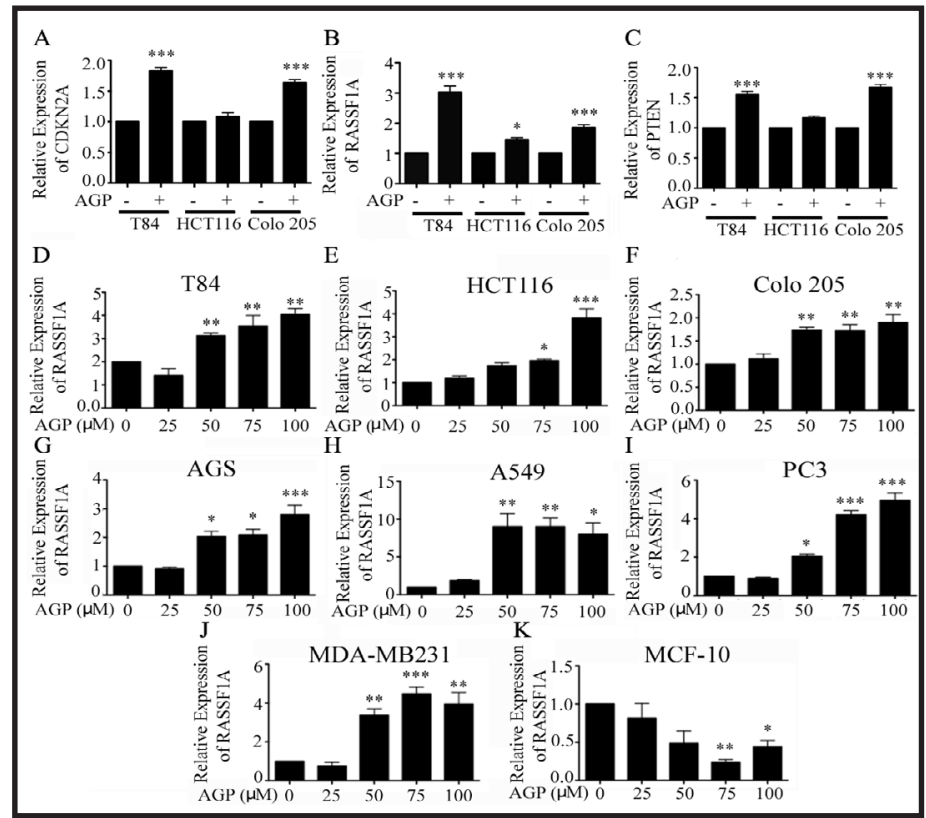

Fig. 1. Andrographolide induces tumor suppressor gene expression in cancer cells. T84, HCT116 and Colo 205 cells were treated with AGP at IC ${ }_{50}(45 \mu \mathrm{M})$ for $48 \mathrm{~h}$ and the transcriptional level of expression for tumor suppressor genes was determined by qRT-PCR for A. CDKN2A, B. RASSF1A, C. PTEN. Bar graphs show quantitative results normalized to GAPDH mRNA levels. Results are from three independent experiments. For dose response determination (D-F) colon cancer cells were treated with increasing concentrations of AGP $(0,25,50$, $75,100 \mu \mathrm{M}$ ) for $48 \mathrm{~h}$. Relative expression of RASSF1A in human colon cancer cells (T84, HCT 116, Colo 205) was determined by qRT-PCR. Dose response determinations were performed on various non-colon cancer cells including G. gastric cancer cells, AGS; H. lung cancer cells, A549; I. prostate cancer cells, PC3; and J. triple negative breast cancer cells, MDA-MB231. K. Relative expression of RASSF1A in noncancer breast epithelial cells (MCF10). Representative quantification from an experiment that was repeated thrice. Statistical significance was determined using one way-ANOVA followed Bonferroni test. $\left({ }^{*} \mathrm{P}<0.05,{ }^{* *} \mathrm{P}<0.01,{ }^{* * *} \mathrm{P}<0.001\right)$. 
cancer. The ability of AGP to induce RASSF1A expression was evaluated in multiple types of cancer cells in a dose dependent manner. AGP induced significantly greater expression in T84 and Colo 205 colon cancer cells and to a lesser extent in HCT 116 cells (Fig. 1D, E, and F). Expression increased at dose ranges above $50 \mu \mathrm{M}$ AGP. Similar results were obtained using gastric (Fig. 1G), lung (Fig. 1H), prostate (Fig. 1I) and triple negative breast (Fig. 1J) cancer cells. Increased expression in these cells required a minimum of $50 \mu$ M AGP. RASSF1A expression in normal breast epithelial cells actually decreased significantly at higher doses of AGP (Fig. 1K). It is probable that a complex form via RASSF1A domain with the histone deacetylase 1 and DNA methyl transferase 1 . However, this needs to be investigated in future.

\section{AGP induced RASSF1A expression promotes cell cycle arrest and apoptosis}

Previous reports have documented the induction of cell cycle arrest and apoptosis in cancer cells treated with AGP [20, 24-26]. We depleted RASSF1A expression with siRNA in T84 and Colo 205 cells to determine the role of RASSF1A in cell cycle arrest and apoptosis following treatment with AGP. RASSF1A siRNA significantly reduced RASSF1A mRNA levels comparable to cells with no AGP treatment (Fig. 2A). Since AGP has been demonstrated to suppress cyclin D1 and cell cycle progression [24] and since RASSF1A can regulate cell cycle progression we also measured cyclin D1 expression in these cells. We observed a significant increase in cyclin D1 mRNA levels in AGP treated T84 cells transfected with RASSF1A siRNA compared to cells transfected with control siRNA (Fig. 2B, $P<0.05)$ or with AGP alone $(P$ $<0.001)$. Similar results were observed for Colo 205 cells $(P<0.001)$. RASSF1A also has the potential to promote apoptosis. We used expression of Bax as a marker for apoptosis signaling. When AGP treated T84 cells were first transfected with RASSF1A siRNA there was a significant decrease in Bax mRNA compared to AGP alone (Fig. $2 \mathrm{C}, P<0.001$ ) or AGP plus control siRNA $(P<0.01)$. Similar results were observed for Colo 205 cells (Fig. 2 C, $P<0.001$ ).

RASSF1A can also promote apoptosis through a pathway involving the activation of LATS1, which phosphorylates the transcription factor YAP [10]. Additionally, RASSF1A can activate MST1 which promotes apoptosis via the Ndr1/2 kinase [13]. Protein analysis on AGP treated colon cancer cells demonstrated a significant increase in phosphorylated YAP in Colo 205 cells (Fig. 2D, $P<0.01$ ). Additional analysis on transcript levels for LATS and MST1 on T84 and Colo 205 cells transfected with RASSF1A siRNA demonstrated that silencing of RASSF1A expression during AGP treatment results in significantly less LATS and MST1 expression compared to cells transfected with control siRNA (see online suppl. material, Suppl. Fig. S2, $P<0.001$ ).

We extended our observations of ex vivo model using human colon cancer organoids treated with AGP. Organoids were treated with increasing doses of AGP (0-90 $\mu \mathrm{m})$. Organoid morphology was evaluated by light microscopy. Organoid structures were observed to condense and dissociate with increasing concentrations of AGP (Fig. 2E) and there was a quantifiable dose-dependent deterioration (Fig. 2F). The dose-dependent cell death was confirmed by the MTT assay (Fig. 2G) [27]. RASSF1A expression was evaluated in this 3D culture model by immunofluorescence. The results indicate significantly greater expression of RASSF1A in AGP treated organoids ( $\mathrm{p}<0.01$ ) compared to untreated cells (Fig. 2H).

AGP induces ER stress, tumor suppressor gene expression, and reduced angiogenesis signaling in tumor tissue in vivo

The efficacy of AGP in limiting colon cancer growth in vivo has been demonstrated in xenogenic mouse models $[8,28,29]$. We employed a short term in vivo AGP treatment analysis to confirm that AGP induces TSG expression in a physiologic setting. We have previously reported that AGP-induced cell death in colon cancer cells requires UPR-mediated ER stress $[16,17]$. Therefore, both TSGs and ER stress markers were evaluated. Tumors were established using HCT 116 cells in the flanks of nude mice and mice were treated for five consecutive days of AGP therapy by either IP or PO administration. Tumor tissue was excised at either $1 \mathrm{~h}$ or $4 \mathrm{~h}$ following the final treatment. Excised tumor tissue was analyzed by qRTPCR. Increased expression of the ER stress-induced unfolded protein response protein 


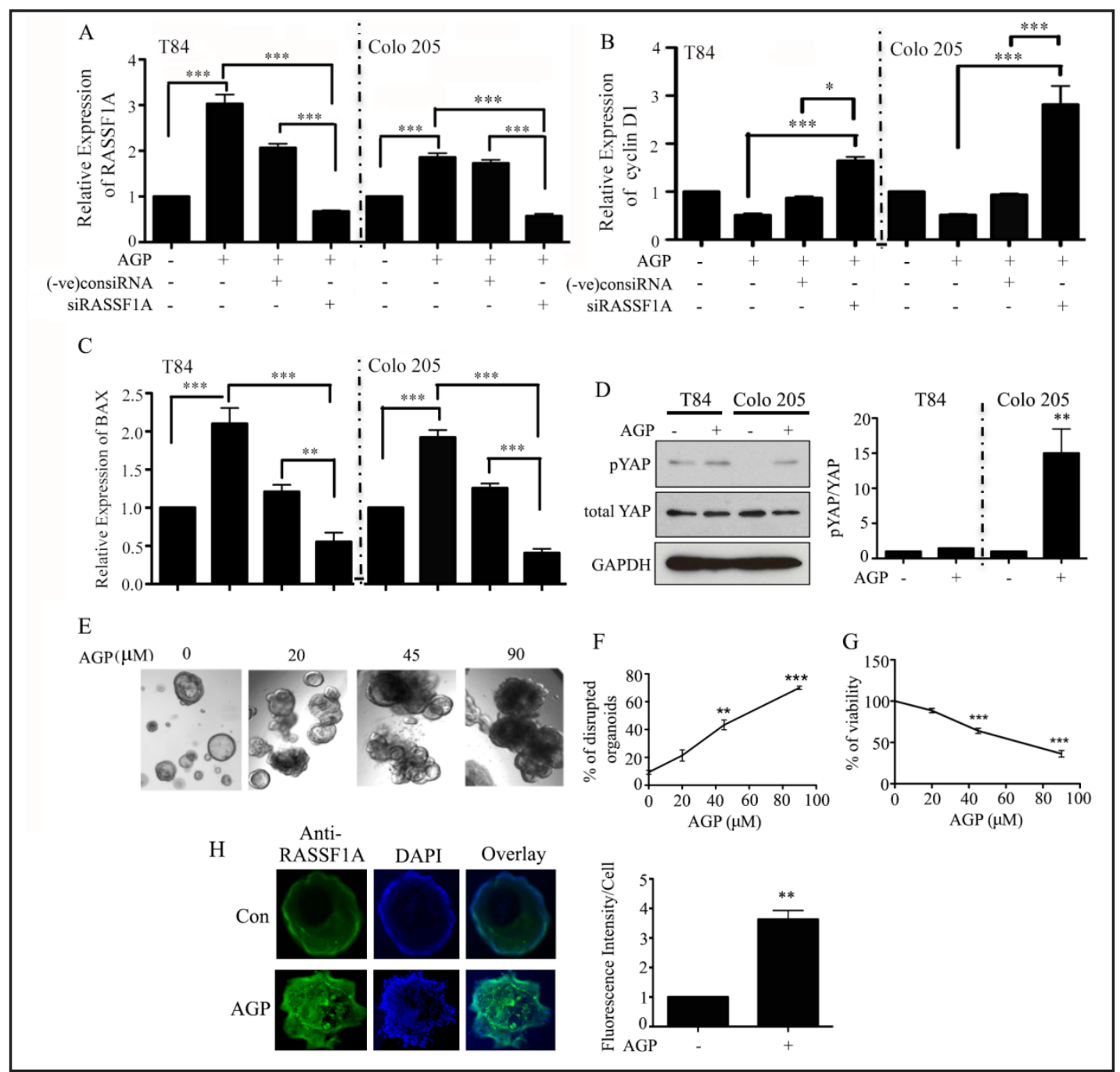

Fig. 2. Andrographolide induced RASSF1A expression promotes cell cycle arrest and apoptosis signaling. T84 (left panels) and Colo 205 cells (right panels) were transfected with RASSF1A siRNA or control siRNA and treated with AGP for $48 \mathrm{~h}$. Cells were evaluated for mRNA expression by qRT-PCR for A. RASSF1A, B. cyclin D1, or C. BAX. D. T84 and Colo 205 cells were treated with or without AGP at IC $_{50}(45 \mu \mathrm{M})$ for $48 \mathrm{~h}$. Cell lysates were analyzed by western blot for phospho-YAP, total YAP and GAPDH expression. Left panel is a representative photograph from an experiment that was repeated three times. Right panel represents quantitative estimations of protein levels determined by densitometry measurements of western blots from three independent experiments after normalization with GAPDH. $\left({ }^{*} \mathrm{P}<0.05\right.$, $\left.{ }^{* *} \mathrm{P}<0.01,{ }^{* * *} \mathrm{P}<0.001\right)$. E. Organoids were treated with AGP for $48 \mathrm{~h}$ and crypt morphology was assessed by light microscopy. F. Enumeration of organoids with altered morphology. At least 100 organoids per well were counted. G. MTT staining of organoids in the presence or absence of AGP. H. RASSF1A staining in organoids in the presence or absence of AGP with FITC-labeled anti-RASSF1A antibody and DAPI. Structures were evaluated by fluorescence microscopy. Data shown are representative for three independent experiments.

IRE-1 $(P<0.05)$ was detected in the $4 \mathrm{~h}$ PO group by compared with untreated tumor (Fig. $3 \mathrm{~A}$ ). No significant expression was found following IP administration or for the $1 \mathrm{~h}$ PO AGP treated group. Upregulation of the ER stress protein GRP-78 was also observed in the $4 \mathrm{~h}$ PO AGP treated group (Fig. 3B; $P<0.05$ ). Tumor suppressor gene expression was also notably increased in the $4 \mathrm{~h}$ PO AGP treatment group including RASSF1A and PTEN (Fig. 3C, $P<0.001$ and Fig. 3D, $P<0.05$ respectively). We also monitored the anti-angiogenic signaling property of AGP since AGP has been reported to limit angiogenesis signaling in some models [30, 31]. 


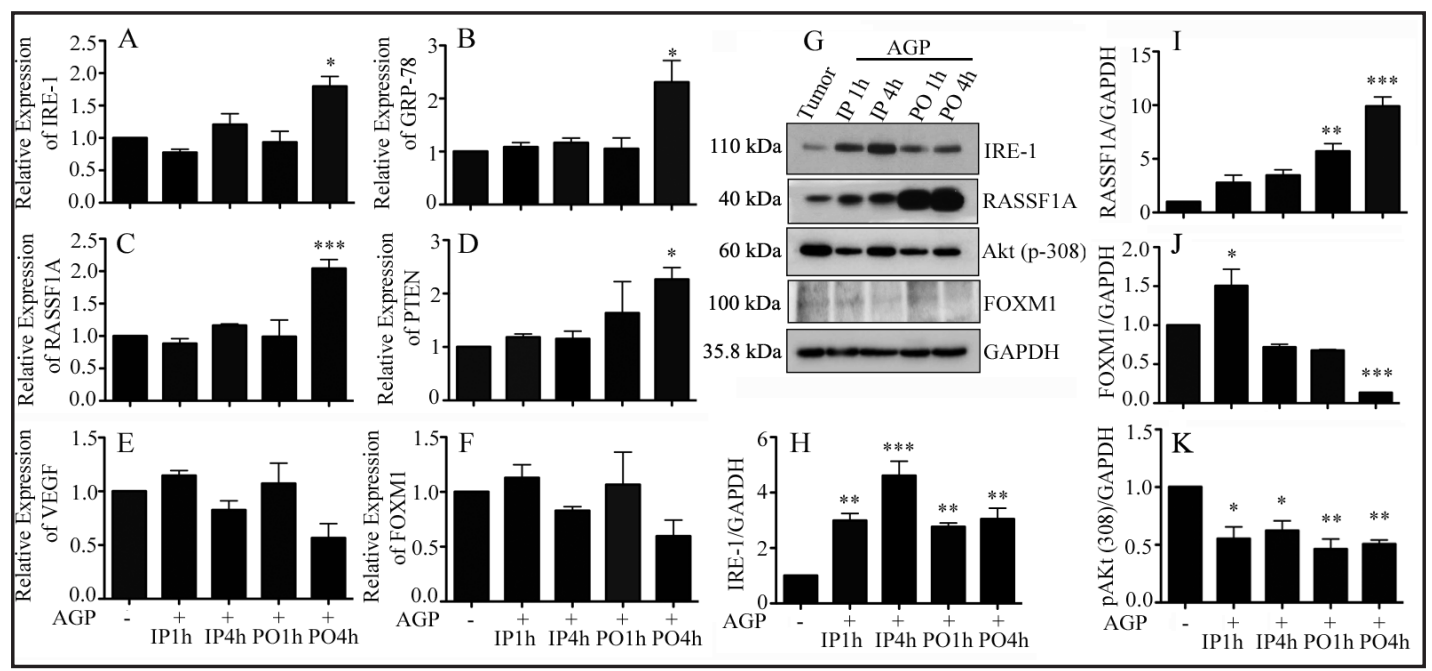

Fig. 3. Andrographolide treatment of colon cancer tumor tissue in vivo induces ER stress, and tumor suppressor genes and suppresses angiogenic signaling. Athymic Balb/c mice were injected subcutaneously with $5 \times 10^{6}$ HCT116 cells in flank for tumor development. Tumor size progressed to $400-500 \mathrm{~mm}^{3}$ and then mice were injected (IP) or orally administered (PO) AGP at a dose of $50 \mathrm{mg} / \mathrm{kg}$ body weight. Transcriptional level of ER stress associated genes (A. IRE-1; B. GRP-78), Tumor suppressor genes (C. RASSF1A and D. PTEN), angiogenesis pathway genes (E. VEGF; F. FOXM1). Bar graphs show quantitative results normalized to GAPDH mRNA levels. G. Western blot analysis of tumor protein extracts treated in vivo with AGP or untreated and quantification of IRE-1 (H), RASSF1A (I), FOXM1 (J) and phospho-Akt (K) by scanning densitometry. One representative example out of 3 is shown. GAPDH was used as a loading control. Statistical significance was determined using one way-ANOVA followed Bonferroni test. $\left({ }^{*} \mathrm{P}<0.05,{ }^{* *} \mathrm{P}<0.01,{ }^{* * *} \mathrm{P}<0.001\right)$.

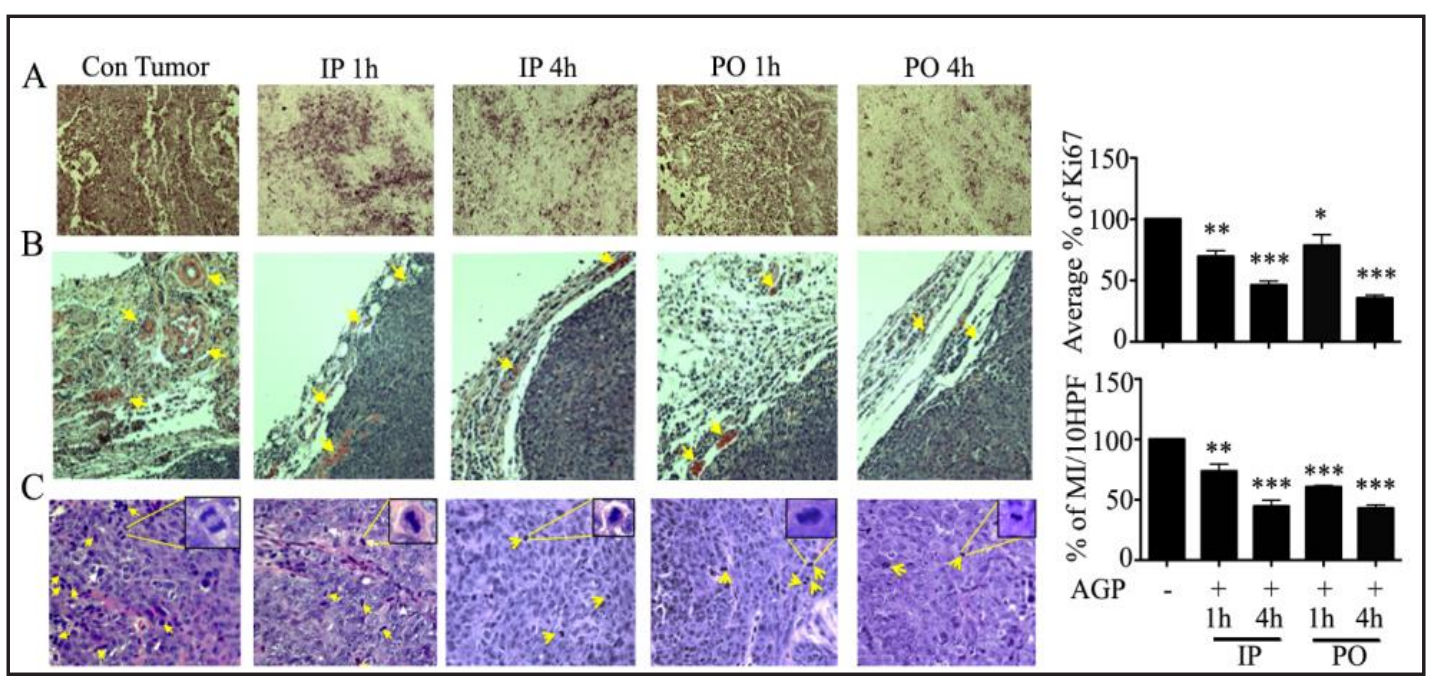

Fig. 4. Proliferation, microvasculature and mitotic index in xenograft tumor tissue from mice treated with andrographolide. A. Immunohistochemistry for Ki67 expression in HCT 116 colon cancer tumor sections with or without AGP treatment (400x). The histogram on the upper right represents the average percentage of Ki67 expression. B. H\&E stained tumor sections identifying the microvascular density in control tumor and following AGP treatment. Arrows indicate microvessels. C. H\&E staining identifying the mitotic index (arrowheads) in control tumor and AGP-treated colon tumor section. The histogram on the lower right represents the percentage of mitotic index from ten representative areas of each tumor. Statistical significance was determined using one way-ANOVA followed Bonferroni test. $\left({ }^{*} \mathrm{P}<0.05,{ }^{* *} \mathrm{P}<0.01\right.$, $* * * \mathrm{P}<0.001)$. 
Levels of VEGF ${ }_{165}$ and FOXM1 in mice harvested $4 \mathrm{~h}$ PO AGP treatment showed decreased expression (Fig. 3E, and 3F respectively). These results were reinforced by protein expression analysis (Fig. 3G) demonstrating significant increases in IRE-1 expression in tumor tissue for all treatments (Fig. 3H), increased RASSF1A expression in mice with AGP PO treatment (Fig. 3I), and significant reductions in FOXM1 expression with AGP 4 h PO treatment (Fig. 3J). Additionally, phospho-Akt levels, an important intermediate in angiogenic signaling was also significantly reduced for all treatments (Fig. 3K).

AGP decreases microvasculature and mitotic index in tumor tissue

Tumor tissues were evaluated for the influence of AGP on the tumor microenvironment and angiogenesis. Cellular proliferation was evaluated by immunohistochemistry for Ki-67 in the presence of absence of AGP treatment. We observed a decrease in the percentage of Ki67 positive cells in all AGP treated groups with the most significant reductions occurring at $4 \mathrm{~h}$ PO or IP treatment (Fig. 4A, P $<0.001$ ). H\&E stained sections were also evaluated and large microvessels were enumerated in five vessel-rich microscopic fields per tumor. AGP treatment at $4 \mathrm{~h}$ PO or IP reduced the size and number of the microvessels (Fig. 4B). The mitotic index of treated or untreated tumor tissues was also measured. We found the reduction in mitotic index to correlate well with the decrease in microvessels in AGP treated tissues, particularly those tumor with $4 \mathrm{~h}$ PO and IP treatment (Fig. 4C, $P<0.001$ ).

AGP induced RASSF1A expression is dependent on ER stress

Our previous studies documented a critical role for ER stress in AGP anti-cancer activity $[16,17]$ and our in vivo analysis demonstrated increased expression of ER stress markers and RASSF1A following AGP treatment (Fig. 3). Therefore, cancer cells were treated with the ER stress inhibitor 4-PBA to determine the importance of ER stress on AGP induced RASSF1A expression. AGP significantly increased RASSF1A protein expression in T84 cells as previously demonstrated for transcriptional analysis (Fig. 1B) but levels returned to baseline in the presence of 4-PBA (Fig. 5A). Similar results were observed in HCT 116 cells (Fig. 5B, $P<0.001)$. Although differences in protein expression were not as stark, AGP treatment did increase RASSF1A levels compared to untreated controls $(P<0.001)$ whereas cells treated with both 4-PBA and AGP did not. Results for Colo 205 cells were less conclusive as 4-PBA failed to reduce protein expression of RASSF1A (Fig. 5C). Analysis performed on T84 cells transfected with siRNA for the ER stress gene IRE-1 demonstrated expression of RASSF1A to be one third that in cells transfected with control siRNA prior to AGP treatment (Fig. 5D, $\mathrm{P}<0.05)$.

\section{PTEN expression promotes RASSF1A expression in AGP treated cells}

Expression of the PTEN tumor suppressor gene was also increased in colon cancer cells (Fig. 1C). PTEN serves as a negative regulator of PI3K/Akt signaling in the survival pathway. We transfected colon cancer cells with PTEN siRNA to determine if AGP induced RASSF1A expression is dependent on PTEN activity. Transfection with PTEN siRNA resulted in significantly reduced PTEN mRNA levels in AGP treated T84 and Colo 205 cells compared to AGP treatment of cells transfected with control siRNA (Fig. 5E, $P<0.001$ ). PTEN levels remained low at both 24 and $48 \mathrm{~h}$. Analysis of RASSF1A expression in these cells showed significant reductions in T84 cells $(\mathrm{P}<0.001)$ and Colo 205 cells $(\mathrm{P}<0.01)$ at $24 \mathrm{~h}$ and 48 $\mathrm{h}$ respectively compared with cells transfected with control siRNA. These data suggest that AGP induced PTEN expression can help regulate RASSF1A in colon cancer cells.

AGP downregulates angiogenic signaling molecules in colon cancer cells

Angiogenesis signaling proteins were suppressed in tumor tissue of AGP treated mice (Fig. 3). We therefore investigated the angiogenic molecule expression in AGP treated colon cancer cells in vitro. Analysis using qRT-PCR demonstrated greater than 50\% reduction of VEGF Vex $_{165}$ expression in T84 and Colo 205 cells following AGP treatment for $48 \mathrm{~h}$ (Fig. 6A, $P<0.001$ ). Similar results were observed for $\mathrm{VEGF}_{189}$ in Colo 205 cells (Fig. 6B, $P<$ 
0.001). Thrombospondin 1 (Tsp-1) and Tsp-2 are potent endogenous inhibitors of angiogenesis with Tsp- 2 more closely associated with inhibition of angiogenesis and metastasis of colon cancer [22, 32]. As shown in Fig. 6C, the Tsp-2 mRNA level was significantly upregulated in AGP treated Colo 205 cells $(P<0.01)$ and T84 cells $(P<0.05)$ compared unstimulated control cells. TSP-1 levels however did not change significantly in either cell line (data not shown). The VEGF receptors were also assessed in these cells lines by but little change was noted in T84 cells until expression of VEGFR2 dropped significantly at $48 \mathrm{~h}$ (Fig. 6D). Colo 205 cells however displayed pronounced increases in expression for both VEGFR1 and VEGFR2 at 8 and $24 \mathrm{~h}$ of stimulation but similar to the T84 cells the expression levels were reduced below control levels.

AGP-induced suppression ofangiogenesis signaling is ER stress dependent

Our results demonstrate that AGPinduced RASSF1A expression is dependent on ER stress (Fig. 5). We analyzed AGP treated cells using chemical or genetic blocking of ER stress proteins to determine their role in suppression of angiogenesis signaling. Blocking ER stress with 4-PBA failed to increase VEGFR expression (see online suppl. material, Suppl. Fig. S3A). We have reported that AGP associated ER stress occurs primarily through the activation of IRE-1 and leads to apoptotic colon cancer cell death [16]. Therefore we evaluated the role of IRE-1 in VEGFR expression. Overexpression of IRE-1 was sufficient to suppress both VEGFR1 and VEGFR2 at $48 \mathrm{~h}$ even in the absence of AGP treatment (see online suppl. material, Suppl. Fig. S3B, $P<$ $0.001)$. We further tested to role of IRE-1 by transfecting colon cancer cells with IRE1 siRNA and monitoring the expression of pro and anti- angiogenic molecules. Little effect was noted on VEGF expression (Fig. $6 \mathrm{E}$ and F). As expected, AGP treatment reduced the expression of FOXM1, Fox03, and PTTG1. Transfection of T84 prior to AGP treatment significantly increased the expression of these proteins comparable to, or greater than, expression levels in controls cells (Fig. 6G, H, and I). Transfection with control siRNA failed to reverse the

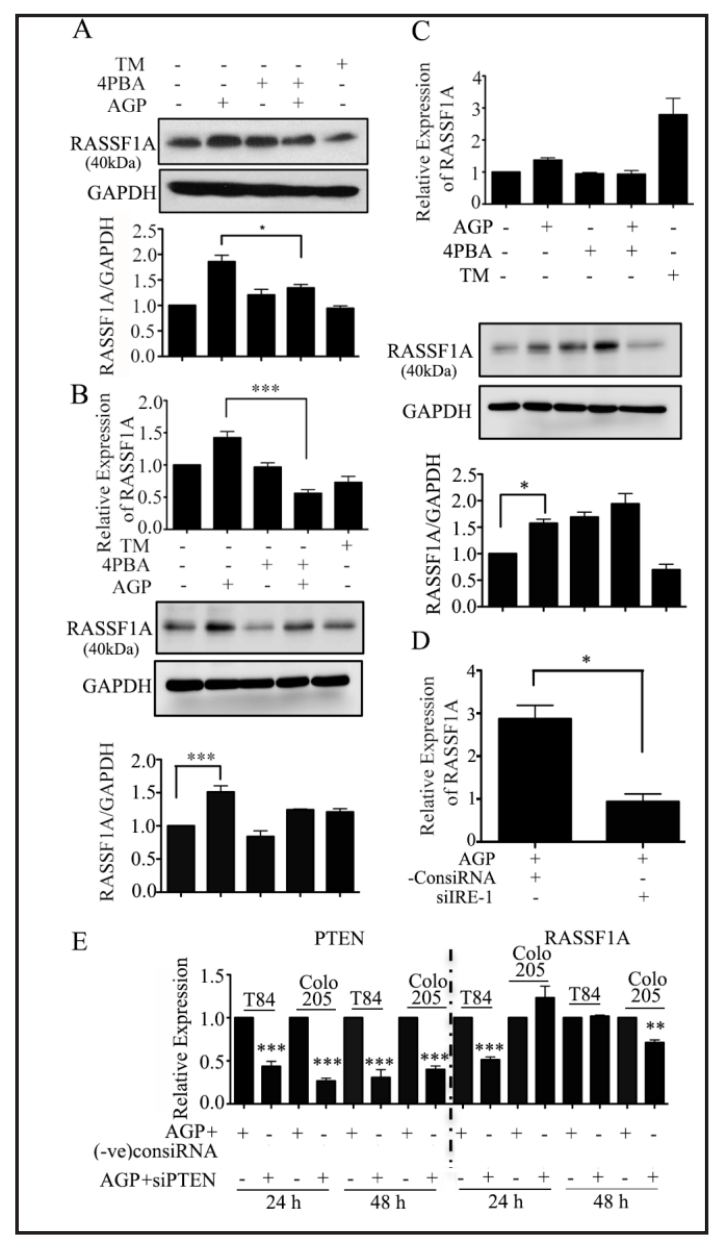

Fig. 5. Andrographolide induced RASSF1A expression is dependent on ER stress and PTEN expression. Colon cancer cells were treated with AGP IC $_{50}$ in the presence or absence of 4PBA for $48 \mathrm{~h}$ and then lysed. Protein expression was determined by western blot for RASSF1A. Densitometry analysis was performed and expression was normalized against GAPDH expression to demonstrate reduced expression of RASSF1A in the presence of $4 \mathrm{PBA}$ in A. T84 cells, B. HCT 116 cells, and C. Colo 205 cells. Results are one representative experiment out of three. Cells were also evaluated for RASSF1A mRNA expression by qRT-PCR in HCT 116 cells (B. upper panel) and in Colo 205 cells (C. upper panel). D. T84 cells were transfected with IRE-1 siRNA or control siRNA and treated with AGP for $48 \mathrm{~h}$ and evaluated for RASSF1A mRNA expression by qRT-PCR. E. T84 and Colo 205 cells were transfected with PTEN siRNA or control siRNA and treated with AGP for $24 \mathrm{~h}$ and $48 \mathrm{~h}$. Cells were evaluated for mRNA expression by qRT-PCR for PTEN (left panel) and RASSF1A (right panel). Statistical significance was determined using one way-ANOVA followed Bonferroni test. $\left({ }^{*} \mathrm{P}<0.05\right.$, ${ }^{* *} \mathrm{P}<0.01,{ }^{* * *} \mathrm{P}<0.001$ ). 


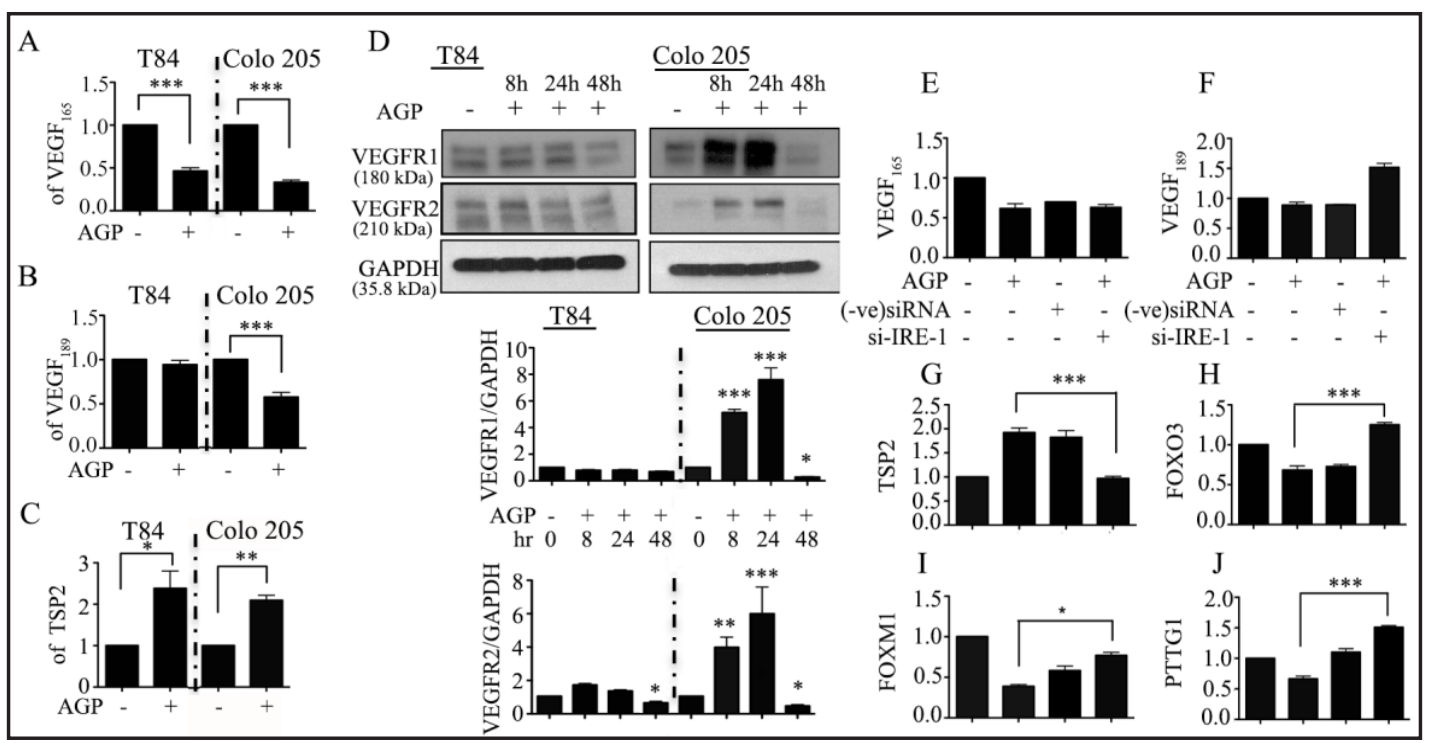

Fig. 6. Andrographolide downregulates angiogenic signaling molecules in colon cancer cells. T84 and Colo 205 cells were analyzed for A. $\mathrm{VEGF}_{165}, \mathrm{~B} . \mathrm{VEGF}_{189}$, and C. TSP2 mRNA expression in the presence or absence of AGP. D. T84 and Colo 205 cells were treated with AGP IC Fi $_{5}$ at $0 \mathrm{~h}, 8 \mathrm{~h}, 24 \mathrm{~h}$ or $48 \mathrm{~h}$ and cell lysates were analyzed by western blot. Photo image is representative of VEGFR1 and VEGFR2 expression. Quantification of the VEGFR1 and VEGFR2 expression was determined by scanning densitometry. E - J. T84 cells were transfected with IRE-1 siRNA or control siRNA and treated with AGP for 48 h. Cells were evaluated for mRNA expression by qRT-PCR for E. VEGF ${ }_{165}$, F. VEGF $_{189}$, G. TSP2, H. FOXO3, I. FOXM1 and J. PTTG1. Statistical significance was determined using one way-ANOVA followed Bonferroni test. $\left({ }^{*} \mathrm{P}<0.05\right.$, $\left.{ }^{* *} \mathrm{P}<0.01,{ }^{* * *} \mathrm{P}<0.001\right)$.

effects of AGP treatment. Conversely, whereas AGP treatment increased expression of the angiogenesis inhibitor Tsp2, transfection with IRE-1 siRNA reduced expression levels to those below background levels (Fig. 6, $P<0.001$ ). These results indicate that AGP induced anti-angiogenesis is mediated via ER stress and in particular the IRE-1 activation pathway. The ability of AGP to suppress angiogenesis signaling is consistent with the reduced vessel formation we observed in treated tumor tissue (Fig. 4), but is confirmed by the ability of AGP to limit endothelial cell viability when tested in vitro (see online suppl. material, Suppl. Fig. S4).

Exogenous VEGF restores Akt phosphorylation and suppresses RASSF1A expression in AGP treated cells

Akt activation stabilize DNA methyltransferase which contributes to the silencing of RASSF1A [33, 34]. Since AGP treatment suppressed angiogenesis signaling including Akt phosphorylation in vivo (Fig. 3) we tested the relationship between angiogenesis signaling and RASSF1A expression. Colon cancer cells were treated with AGP in the presence or absence of exogenous $\mathrm{VEGF}_{165}$. The expression of the VEGF receptors was evaluated to determine if exogenous VEGF ${ }_{165}$ blocks AGP suppression of angiogenesis signaling (Fig. 7A). Immunoblot analysis for the VEGFR1 and VEGFR2 demonstrated a significant increase in colon cancer cells stimulated with exogenous $\mathrm{VEGF}_{165}$ compared with unstimulated cells although the addition of $\mathrm{VEGF}_{165}$ to AGP treated cells failed to prevent the suppression of these receptors (Fig. 7B). The addition of $\mathrm{VEGF}_{165}$ did however, promote the phosphorylation of Akt, and when $\mathrm{VEGF}_{165}$ combined with AGP Akt activation remained significantly greater than cells treated with AGP alone (Fig. 7C). These data indicated $\mathrm{VEG}_{165}$ treatment supersedes the effects of AGP with respect to downstream angiogenesis signaling events. The addition of $\mathrm{VEGF}_{165}$ also prevented the upregulation of RASSF1A expression by AGP (Fig. 7D). Whereas AGP alone induced significantly greater levels of RASSF1A compared to unstimulated cells 
$(P<0.001)$, co-treatment with $\mathrm{VEGF}_{165}$ and AGP resulted in RASSF1A expression not appreciably different from unstimulated cells.

\section{Discussion}

AGP, isolated from Andrographis paniculata, has been shown to possess cytotoxic activity and induce cell cycle arrest in multiple types of human cancer cells including epidermoid leukemia and lymphocytic leukemia, as well as liver, breast, colon, prostate, and lung cancer cell lines. There have been several studies to explore translational activities of AGP. Some of the clinical trials are ongoing for acute bronchitis, tonsillitis (https://clinicaltrials.gov/ ct2/show/NCT03132623). Additional clinical study has performed (http://ascopubs. org / d o i / a b s / 10.1200 / JC0.2017.35.4_suppl.TPS819) to implicate AGP for colon cancer treatment.

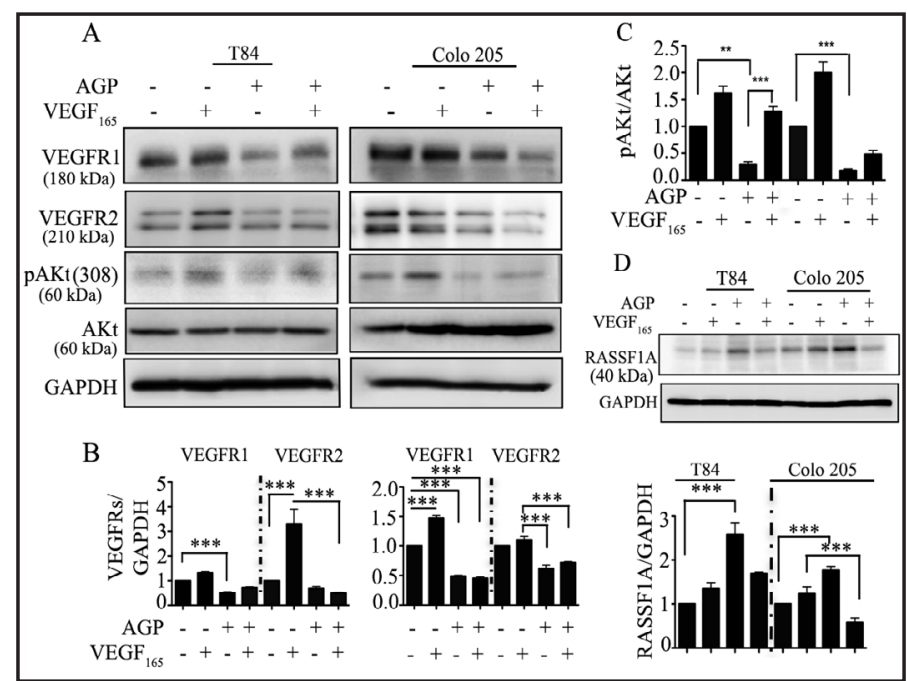

Fig. 7. Exogenous VEGF restores Akt phosphorylation and suppresses RASSF1A expression in AGP treated cells. T84 and Colo 205 cells were stimulated with $\mathrm{VEGF}_{165}(10 \mathrm{ng} /$ well) for $10 \mathrm{mins}$ and then treated with AGP $\left(\mathrm{IC}_{50}\right)$ for $48 \mathrm{~h}$. A. Cell lysates were monitored by western blot for VEGFR1, VEGFR2, phospho Akt, total Akt and GAPDH as indicated. B. VEGFR1 and VEGFR2 expression from western blotting was quantified by scanning densitometry for T84 (left panel), Colo 205 cells (Middle panel). C. The ratio of phosphoAkt to total Akt was determined by scanning densitometry in T84 (left panel) Colo 205 cells (right panel). D. RASSF1A expression was also determined by immunoblot analysis in AGP treated cells with prior incubation with VEGF. Results were quantified by scanning densitometry and normalized against GAPDH expression in T84 (left panel) and Colo 205 cells (right panel). $\left({ }^{*} \mathrm{P}<0.05,{ }^{* *} \mathrm{P}<0.01\right.$, $\left.{ }^{* * *} \mathrm{P}<0.001\right)$.

\section{To better understand}

the molecular mechanism of AGP in colon cancer cell death and to extend our previous observations we used AGP in this study. The previous observations are: 1) AGP induces cell death in CRC cells and this activity is dependent on the activation of IRE-1, an ER-stress marker. 2) However, in the subsequent study we found, AGP induces ER stress leading to apoptosis through the induction of Reactive Oxygen Species (ROS) and play an important role in down-regulating cell cycle progression and cell survival pathways as well. 3) In addition, we have also demonstrated that AGP downregulates the Akt/mTOR survival pathway in this model [17]. In our study, we also observed inactivation of $\mathrm{NF}-\mathrm{k} \beta$ in andrographolide treated colon cancer cells (data not shown) similar to the previously reported [25]. In this article, we are interested in monitoring the RASSF1A regulation by suppression of angiogenesis signaling and Akt activation. In the present study, we have demonstrated if AGP regulates tumor suppressor gene expression, we first monitored different tumor suppressor gene expression which are involved in colon cancer progression, we found RASSF1A, PTEN and CDKN2A are upregulated by AGP treatment in colon cancer cells (Fig.1).

Our results demonstrate that AGP significantly upregulates expression of the epigenetically silenced TSG RASSF1A, as well as PTEN and CDKN2A. Increased expression was noted in vitro and in vivo in AGP treated mice harboring HCT 116 tumors. Little is known about the potential of AGP to induce TSGs. AGP, alone or combined with cisplatin, has been shown to activate $\mathrm{p} 53$ [25, 35]. Additionally, AGP upregulates the HLJ1 TSG via Jun $B$ activation in non-small cell lung cancer cells [36]. This is the first report documenting the induction of RASSF1A, PTEN, and CDKN2A expression by AGP. 
The RASSF1A activity observed in our model is consistent with functions described in other systems [2,7]. It has been demonstrated to influence cell survival by several distinct mechanisms. Signaling through mitogenic stimuli such as growth factor receptors is associated with blocking cell cycle progression by indirectly regulating at Cyclin D and Cyclin A activities resulting in arrest at either the G1/S or G2/M phases [7, 37-39]. Signaling events associated with death receptor activity can also involve RASSF1A and these events promote apoptosis signaling. RASSF1A has been shown to activate Bax, but has also been shown to activate MST1 which leads to apoptosis $[9,12]$. It can also activate MST2 leading to LATS and YAP activation which also promote the transcription of proapoptosis factors [10]. In our model, RASSF1A depletion using siRNA during AGP treatment of cancer cells resulted in reduced expression of Bax, but of MST1 and LATS as well, suggesting the involvement of several members of the canonical hippo pathway during AGP treatment.

RASSF1A is regulated through epigenetic modifications. Akt activation induces DNA methyltransferase (DNMT) to methylate the RASSF1A gene which inhibits its expression. Akt activation and function is complex but growth factor receptor signaling in which PIP $_{2}$ is converted to $\mathrm{PIP}_{3}$ by PI3K contributes to Akt activation. The conversion of $\mathrm{PIP}_{2}$ to $\mathrm{PIP}_{3}^{2}$ is blocked by the tumor suppressor gene PTEN [40] which we observed to be significantly upregulated by AGP treatment. Depletion of PTEN during AGP treatment resulted in a $50 \%$ reduction of RASSF1A expression. Therefore, upregulation of PTEN expression is an important aspect of AGP activity with respect to increasing RASSF1A expression.

Increased expression of RASSF1A has also been described in prostate cancer cells treated with the plant biomaterial mahanine $[2,41]$. Treatment of the cells with mahanine resulted in proteasome degradation of DNMT resulting in increased RASSF1A expression. Mahanine was shown to inhibit PDK1, which consequently results in reduced activation of Akt and then degradation of DNMT. Therefore, both mahanine and AGP act on the same pathway, although on different proteins, to effectively limit Akt activity which translates to increased RASSF1A expression.

We also observed AGP to suppress angiogenesis signaling. This is consistent with several other reports in which AGP was shown limit angiogenesis by targeting the miR21-5p regulatory network and Notch1 pathway that drives CD133 expression to control melanoma growth and angiogenesis $[30,31]$. In our model, AGP was shown to suppress the expression of VEGF receptors, FoxO3a and FOXM1 and to increase expression of the antiangiogenesis factor TSP-2. Many of these observations were observed in our in vivo tumor model as well. Microvascular count (MVC) for the quantification of tumor angiogenesis is a measure of tumor staging, as well as the potential for invasiveness and metastasis [42, 43]. In the present study we found that AGP significantly reduced microvessel size and number in tumor tissue treated with AGP as further evidence for AGPs anti-angiogenesis activity.

It was of particular interest that AGP suppressed angiogenesis signaling activity since it includes reduced Akt activation. Activated Akt is important for stabilizing DNMT, which in turn suppresses RASSF1A expression. Thus, reduced Akt activation would result in increased RASSF1A. The addition of exogenous VEGF was sufficient to prevent AGP from suppressing angiogenesis signaling and this resulted in significantly less RASSF1A expression compared to cells treated with AGP in the absence of VEGF. To the best of our knowledge, this is the first demonstration that RASSF1A expression is linked to angiogenesis signaling activity as illustrated (see online suppl. material) in Suppl. Fig. S5. Therefore, in our model of AGP treatment, both PTEN expression and suppression of angiogenesis signaling contributed to increased RASSF1A expression.

In summary, these results demonstrate for the first time that AGP induces RASSF1A expression in colon cancer cells in vitro and in vivo and this expression is ER stress dependent. These results also demonstrate AGP has an anti-angiogenic property in colon cancer cells and that RASSF1A expression is dependent on angiogenic signaling events. The mechanism by which AGP influences the suppression of angiogenic signaling is not clear. Therefore, further investigation on AGP will be necessary to delineate its molecular interactions with VEGFR and other key elements in the angiogenesis pathway. 


\section{Cellular Physiology Cell Physiol Biochem 2018;48:1259-1273 \begin{tabular}{l|l} 
and Biochemistry Published online: $10.1159 / 000492012$ & $\begin{array}{l}\text { C) } 2018 \text { The Author(s). Published by S. Karger AG, Basel } \\
\text { www.karger.com/cpb }\end{array}$
\end{tabular}}

Blanchard et al.: RASSF1A Regulation by Angiogenic Signal

\section{Acknowledgements}

We wish to thank the Department of Pediatrics at the University of Maryland School of Medicine for support. We also thank Drs. Sandra Mooney and Alex Medina (Department of Pediatrics) for the use of their respective fluorescence microscopes and imaging facilities.

\section{Disclosure Statement}

The authors declare that they have no competing interests.

\section{References}

1 Goyette MC, Cho K, Fasching CL, Levy DB, Kinzler KW, Paraskeva C, Vogelstein B, Stanbridge EJ: Progression of colorectal cancer is associated with multiple tumor suppressor gene defects but inhibition of tumorigenicity is accomplished by correction of any single defect via chromosome transfer. Mol Cell Biol 1992;12:1387-1395.

-2 Jagadeesh S, Sinha S, Pal BC, Bhattacharya S, Banerjee PP: Mahanine reverses an epigenetically silenced tumor suppressor gene RASSF1A in human prostate cancer cells. Biochem Biophys Res Commun 2007;362:212-217.

3 van Engeland M, Roemen GM, Brink M, Pachen MM, Weijenberg MP, de Bruine AP, Arends JW, van den Brandt PA, de Goeij AF, Herman JG: K-ras mutations and RASSF1A promoter methylation in colorectal cancer. Oncogene 2002;21:3792-3795.

4 Sakamoto N, Terai T, Ajioka Y, Abe S, Kobayasi O, Hirai S, Hino O, Watanabe H, Sato N, Shimoda T, Fujii H: Frequent hypermethylation of RASSF1A in early flat-type colorectal tumors. Oncogene 2004;23:89008907.

-5 Yoon JH, Dammann R, Pfeifer GP: Hypermethylation of the CpG island of the RASSF1A gene in ovarian and renal cell carcinomas. Int J Cancer 2001;94:212-217.

6 Agathanggelou A, Bieche I, Ahmed-Choudhury J, Nicke B, Dammann R, Baksh S, Gao B, Minna JD, Downward J, Maher ER, Latif F: Identification of novel gene expression targets for the Ras association domain family 1 (RASSF1A) tumor suppressor gene in non-small cell lung cancer and neuroblastoma. Cancer Res 2003; 63:5344-5351.

7 Shivakumar L, Minna J, Sakamaki T, Pestell R, White MA: The RASSF1A tumor suppressor blocks cell cycle progression and inhibits cyclin D1 accumulation. Mol Cell Biol 2002; 22:4309-4318.

-8 Rajagopal S, Kumar RA, Deevi DS, Satyanarayana C, Rajagopalan R: Andrographolide, a potential cancer therapeutic agent isolated from Andrographis paniculata. J Exp Ther Oncol 2003;3:147-158.

-9 Baksh S, Tommasi S, Fenton S, Yu VC, Martins LM, Pfeifer GP, Latif F, Downward J, Neel BG: The tumor suppressor RASSF1A and MAP-1 link death receptor signaling to Bax conformational change and cell death. Mol Cell 2005;18:637-650.

-10 Donninger H, Allen N, Henson A, Pogue J, Williams A, Gordon L, Kassler S, Dunwell T, Latif F, Clark GJ: Salvador protein is a tumor suppressor effector of RASSF1A with hippo pathway-independent functions. J Biol Chem 2011;286:18483-18491.

11 Foley CJ, Freedman H, Choo SL, Onyskiw C, Fu NY, Yu VC, Tuszynski J, Pratt JC, Baksh S: Dynamics of RASSF1A/MOAP-1 association with death receptors. Mol Cell Biol 2008;28:4520-4535.

12 Oh HJ, Lee KK, Song SJ, Jin MS, Song MS, Lee JH, Im CR, Lee JO, Yonehara S, Lim DS: Role of the tumor suppressor RASSF1A in Mst1-mediated apoptosis. Cancer Res 2006;66:2562-2569.

13 Vichalkovski A, Gresko E, Cornils H, Hergovich A, Schmitz D, Hemmings BA: NDR kinase is activated by RASSF1A/MST1 in response to Fas receptor stimulation and promotes apoptosis. Curr Biol 2008;18:18891895.

14 Lassoued W, Murphy D, Tsai J, Oueslati R, Thurston G, Lee WM: Effect of VEGF and VEGF Trap on vascular endothelial cell signaling in tumors. Cancer Biol Ther 2010;10:1326-1333. 


\section{Cellular Physiology Cell Physiol Biochem 2018;48:1259-1273 \begin{tabular}{l|l} 
and Biochemistry Published online: July 25, 2018 & $\begin{array}{l}\text { (c) } 2018 \text { The Author(s). Published by S. Karger AG, Basel } \\
\text { www.karger.com/cpb }\end{array}$
\end{tabular}}

15 Suzuma K, Naruse K, Suzuma I, Takahara N, Ueki K, Aiello LP, King GL: Vascular endothelial growth factor induces expression of connective tissue growth factor via KDR, Flt1, and phosphatidylinositol 3-kinaseAkt-dependent pathways in retinal vascular cells. J Biol Chem 2000;275:40725-40731.

-16 Banerjee A, Ahmed H, Yang P, Czinn SJ, Blanchard TG: Endoplasmic reticulum stress and IRE-1 signaling cause apoptosis in colon cancer cells in response to andrographolide treatment. Oncotarget 2016;7(27):41432-41444.

17 Banerjee A, Banerjee V, Czinn S, Blanchard T: Increased reactive oxygen species levels cause ER stress and cytotoxicity in andrographolide treated colon cancer cells. Oncotarget 2017;8:26142-26153.

18 Sato T, Stange DE, Ferrante M, Vries RG, Van Es JH, Van den Brink S, Van Houdt WJ, Pronk A, Van Gorp J, Siersema PD, Clevers H: Long-term expansion of epithelial organoids from human colon, adenoma, adenocarcinoma, and Barrett's epithelium. Gastroenterology 2011;141:1762-1772.

19 Fisch AS, Yerges-Armstrong LM, Backman JD, Wang H, Donnelly P, Ryan KA, Parihar A, Pavlovich MA, Mitchell BD, O'Connell JR, Herzog W, Harman CR, Wren JD, Lewis JP: Genetic Variation in the Platelet Endothelial Aggregation Receptor 1 Gene Results in Endothelial Dysfunction. PLoS One 2015;10:e0138795.

20 Kim TG, Hwi KK, Hung CS: Morphological and biochemical changes of andrographolide-induced cell death in human prostatic adenocarcinoma PC-3 cells. In vivo 2005;19:551-557.

21 Banerjee A, Basu M, Blanchard TG, Chintalacharuvu SR, Guang W, Lillehoj EP, Czinn SJ: Early Molecular Events in Murine Gastric Epithelial Cells Mediated by Helicobacter pylori CagA. Helicobacter 2016;21:395404.

22 Banerjee A, Lang JY, Hung MC, Sengupta K, Banerjee SK, Baksi K, Banerjee DK: Unfolded protein response is required in nu/nu mice microvasculature for treating breast tumor with tunicamycin. J Biol Chem 2011;286:29127-29138.

23 Shiu J, Piazuelo MB, Ding H, Czinn SJ, Drakes ML, Banerjee A, Basappa N, Kobayashi KS, Fricke WF, Blanchard TG: Gastric LTi cells promote lymphoid follicle formation but are limited by IRAK-M and do not alter microbial growth. Mucosal Immunol 2015;8:1047-1059.

24 Shi MD, Lin HH, Lee YC, Chao JK, Lin RA, Chen JH: Inhibition of cell-cycle progression in human colorectal carcinoma Lovo cells by andrographolide. Chem Biol Interact 2008;174:201-210.

25 Zhou J, Lu GD, Ong CS, Ong CN, Shen HM: Andrographolide sensitizes cancer cells to TRAIL-induced apoptosis via p53-mediated death receptor 4 up-regulation. Mol Cancer Ther 2008;7:2170-2180.

26 Zhou J, Zhang S, Ong CN, Shen HM: Critical role of pro-apoptotic Bcl-2 family members in andrographolideinduced apoptosis in human cancer cells. Biochem Pharmacol 2006;72:132-144.

27 Boehnke K, Iversen PW, Schumacher D, Lallena MJ, Haro R, Amat J, Haybaeck J, Liebs S, Lange M, Schafer R, Regenbrecht CR, Reinhard C, Velasco JA: Assay Establishment and Validation of a High-Throughput Screening Platform for Three-Dimensional Patient-Derived Colon Cancer Organoid Cultures. J Biomol Screen 2016;21:931-941.

28 Lin HH, Shi MD, Tseng HC, Chen JH: Andrographolide sensitizes the cytotoxicity of human colorectal carcinoma cells toward cisplatin via enhancing apoptosis pathways in vitro and in vivo. Toxicol Sci 2014;139:108-120.

29 Wong CC, Lim SH, Sagineedu SR, Lajis NH, Stanslas J: SRJ09, a promising anticancer drug lead: Elucidation of mechanisms of antiproliferative and apoptogenic effects and assessment of in vivo antitumor efficacy. Pharmacol Res 2016;107:66-78.

30 Dai J, Lin Y, Duan Y, Li Z, Zhou D, Chen W, Wang L, Zhang QQ: Andrographolide Inhibits Angiogenesis by Inhibiting the Mir-21-5p/TIMP3 Signaling Pathway. Int J Biol Sci 2017;13:660-668.

-31 Kumar D, Kumar S, Gorain M, Tomar D, Patil HS, Radharani NN, Kumar TV, Patil TV, Thulasiram HV, Kundu GC: Notch1-MAPK Signaling Axis Regulates CD133+ Cancer Stem Cell-Mediated Melanoma Growth and Angiogenesis. J Invest Dermatol 2016;136:2462-2474.

-32 Tokunaga T, Nakamura M, Oshika Y, Abe Y, Ozeki Y, Fukushima Y, Hatanaka H, Sadahiro S, Kijima H, Tsuchida T, Yamazaki H, Tamaoki N, Ueyama Y: Thrombospondin 2 expression is correlated with inhibition of angiogenesis and metastasis of colon cancer. Br J Cancer 1999;79:354-359.

-33 Esteve PO, Chang Y, Samaranayake M, Upadhyay AK, Horton JR, Feehery GR, Cheng X, Pradhan S: A methylation and phosphorylation switch between an adjacent lysine and serine determines human DNMT1 stability. Nat Struct Mol Biol 2011;18:42-48. 


\section{Cellular Physiology Cell Physiol Biochem 2018;48:1259-1273 \begin{tabular}{l|l} 
DOI: 10.1159/000492012 & $\begin{array}{l}\text { O 2018 The Author(s). Published by S. Karger AG, Basel } \\
\text { www.karger.com/cpb }\end{array}$
\end{tabular} \\ Blanchard et al.: RASSF1A Regulation by Angiogenic Signal}

34 Zhang H, He J, Li J, Tian D, Gu L, Zhou M: Methylation of RASSF1A gene promoter is regulated by p53 and DAXX. FASEB J 2013;27:232-242.

-35 Chen S, Hu H, Miao S, Zheng J, Xie Z, Zhao H: Anti-tumor effect of cisplatin in human oral squamous cell carcinoma was enhanced by andrographolide via upregulation of phospho-p53 in vitro and in vivo. Tumour Biol 2017;39:1010428317705330.

-36 Lai YH, Yu SL, Chen HY, Wang CC, Chen HW, Chen JJ: The HLJ1-targeting drug screening identified Chinese herb andrographolide that can suppress tumour growth and invasion in non-small-cell lung cancer. Carcinogenesis 2013;34:1069-1080.

-37 Ahmed-Choudhury J, Agathanggelou A, Fenton SL, Ricketts C, Clark GJ, Maher ER, Latif F: Transcriptional regulation of cyclin A2 by RASSF1A through the enhanced binding of p120E4F to the cyclin A2 promoter. Cancer Res 2005;65:2690-2697.

38 Fenton SL, Dallol A, Agathanggelou A, Hesson L, Ahmed-Choudhury J, Baksh S, Sardet C, Dammann R, Minna JD, Downward J, Minna JD, Downward J, Maher ER, Latif F: Identification of the E1A-regulated transcription factor p120 E4F as an interacting partner of the RASSF1A candidate tumor suppressor gene. Cancer Res 2004;64:102-107.

39 Whang YM, Kim YH, Kim JS, Yoo YD: RASSF1A suppresses the c-Jun-NH2-kinase pathway and inhibits cell cycle progression. Cancer Res 2005;65:3682-3690.

40 Maehama T, Dixon JE: The tumor suppressor, PTEN/MMAC1, dephosphorylates the lipid second messenger, phosphatidylinositol 3, 4,5-trisphosphate. J Biol Chem 1998;273:13375-13378.

41 Agarwal S, Amin KS, Jagadeesh S, Baishay G, Rao PG, Barua NC, Bhattacharya S, Banerjee PP: Mahanine restores RASSF1A expression by down-regulating DNMT1 and DNMT3B in prostate cancer cells. Mol Cancer 2013;12:99.

-42 Faviana P, Boldrini L, Spisni R, Berti P, Galleri D, Biondi R, Camacci T, Materazzi G, Pingitore R, Miccoli P, Fontanini G: Neoangiogenesis in colon cancer: correlation between vascular density, vascular endothelial growth factor (VEGF) and p53 protein expression. Oncol Rep 2002;9:617-620.

43 Sakurai K, Yamada N, Yashiro M, Matsuzaki T, Komatsu M, Ohira M, Miwa A, Hirakawa K: A novel angiogenesis inhibitor, Ki23057, is useful for preventing the progression of colon cancer and the spreading of cancer cells to the liver. Eur J Cancer 2007;43:2612-2620. 